\title{
Hydrogen Sulfide Protects against Chronic Unpredictable Mild Stress-Induced Oxidative Stress in Hippocampus by Upregulation of BDNF-TrkB Pathway
}

\author{
Min Hu, ${ }^{1,2}$ Wei Zou, ${ }^{1}$ Chun-Yan Wang, ${ }^{3}$ Xi Chen, ${ }^{4}$ Hui-Ying Tan, ${ }^{1,2}$ Hai-Ying Zeng, ${ }^{1,2}$ \\ Ping Zhang, ${ }^{1}$ Hong-Feng Gu, ${ }^{2}$ and Xiao-Qing Tang ${ }^{1,2}$ \\ ${ }^{1}$ Department of Neurology, Nanhua Affiliated Hospital, University of South China, Hengyang, Hunan 421001, China \\ ${ }^{2}$ Institute of Neuroscience, Medical College, University of South China, Hengyang, Hunan 421001, China \\ ${ }^{3}$ Department of Pathophysiology, Medical College, University of South China, Hengyang, Hunan 421001, China \\ ${ }^{4}$ Department of Anatomy, Medical College, University of South China, Hengyang, Hunan 421001, China
}

Correspondence should be addressed to Xi Chen; chxi1964@126.com and Xiao-Qing Tang; tangxq-usc@qq.com

Received 5 April 2016; Revised 10 June 2016; Accepted 15 June 2016

Academic Editor: Massimo Collino

Copyright (C) $2016 \mathrm{Min} \mathrm{Hu}$ et al. This is an open access article distributed under the Creative Commons Attribution License, which permits unrestricted use, distribution, and reproduction in any medium, provided the original work is properly cited.

\begin{abstract}
Chronic unpredictable mild stress (CUMS) induces hippocampal oxidative stress. $\mathrm{H}_{2} \mathrm{~S}$ functions as a neuroprotectant against oxidative stress in brain. We have previously shown the upregulatory effect of $\mathrm{H}_{2} \mathrm{~S}$ on BDNF protein expression in the hippocampus of rats. Therefore, we hypothesized that $\mathrm{H}_{2} \mathrm{~S}$ prevents CUMS-generated oxidative stress by upregulation of BDNF-TrkB pathway. We showed that NaHS ( 0.03 or $0.1 \mathrm{mmol} / \mathrm{kg}$ /day) ameliorates the level of hippocampal oxidative stress, including reduced levels of malondialdehyde (MDA) and 4-hydroxy-2-trans-nonenal (4-HNE), as well as increased level of glutathione (GSH) and activity of superoxide dismutase (SOD) in the hippocampus of CUMS-treated rats. We also found that $\mathrm{H}_{2} \mathrm{~S}$ upregulated the level of BDNF and p-TrkB protein in the hippocampus of CUMS rats. Furthermore, inhibition of BDNF signaling by K252a, an inhibitor of the BDNF receptor TrkB, blocked the antioxidant effects of $\mathrm{H}_{2} \mathrm{~S}$ on CUMS-induced hippocampal oxidative stress. These results reveal the inhibitory role of $\mathrm{H}_{2} \mathrm{~S}$ in CUMS-induced hippocampal oxidative stress, which is through upregulation of BDNF/TrkB pathway.
\end{abstract}

\section{Introduction}

Mood and anxiety disorders have been substantially associated with stressful life. They frequently appear early in life events, cause a chronic course, and adversely affect individual's productive life $[1,2]$. Moreover, the current synthetic antistress drugs have low efficacy and even severe adverse-effects. Therefore, understanding the prevention bases of these disorders is crucial. Chronic unpredictable stress (CUMS) is a moderate intensity of stress, which treats experimental animals mainly through long-term and given relatively various mild stressors. Our previous studies reported that CUMS results in damage to the hippocampus [3]. Notably, it has been shown that stress can lead to neuronal atrophy and loss in certain brain structures, mainly in the hippocampus $[1,4]$. In parallel, exogenous stress is also reported to induce neuronal cell death in the hippocampus [5]. Meanwhile, elevated hippocampal oxidative stress plays a vital role for neurotoxicity and neuronal death toward the progression of CUMS-treated rats $[6,7]$.

Hydrogen sulfide $\left(\mathrm{H}_{2} \mathrm{~S}\right)$, the third gaseous mediator [8], has been recognized to play crucial important roles in physiological functions of central nervous system $[9,10]$. $\mathrm{H}_{2} \mathrm{~S}$ enhances the induction of long-term potentiation (LTP) in the hippocampus $[11,12]$ and regulates intracellular $\mathrm{Ca}^{2+}$ waves in neurons $[13,14]$, which indicates that $\mathrm{H}_{2} \mathrm{~S}$ is a neuromodulator. Interesting, the critical role of $\mathrm{H}_{2} \mathrm{~S}$ in suppressing oxidative stress has been confirmed $[15,16]$. In addition, recent report demonstrated that exogenous $\mathrm{H}_{2} \mathrm{~S}$ alleviated the oxidative stress-induced rat hippocampal damage through its antioxidant effects [17]. Moreover, we have previously provided compelling evidence that CUMS 
induced the imbalance of proportion to endogenous $\mathrm{H}_{2} \mathrm{~S}$ in hippocampus [18]. Thus, we speculated that $\mathrm{H}_{2} \mathrm{~S}$ attenuates oxidative stress in hippocampus of CUMS-treated rats.

Brain-derived neurotrophins factor (BDNF) is a member of the neurotrophins family, which exerts its roles via its high affinity receptor tyrosine protein kinase B (TrkB) [19]. BDNF and its receptor TrkB, which are widely and abundantly expressed throughout in the CNS, activate various intracellular signaling pathways associated with the neuroprotective effects [20]. Numerous studies have also documented that stress significantly decreases BDNF mRNA expression in the hippocampus $[4,21]$. Recent studies reported that stress decreases the expression of BDNF in the frontal cortex and hippocampus of rodents [22]. Meanwhile, it is conceivable that BDNF downregulates the ethanol-induced cellular oxidative stress and apoptosis in developing hypothalamic neuronal cells [23]. Furthermore, our previous studies have shown that BDNF-TrkB pathway mediates the protective role of $\mathrm{H}_{2} \mathrm{~S}$ against FA-induced oxidative damage in PC12 cells [24]. Therefore, we will investigate whether the protection of $\mathrm{H}_{2} \mathrm{~S}$ against CUMS-induced hippocampal oxidative stress is also via $\mathrm{BDNF} / \mathrm{TrkB}$ pathway.

In the current study, our results identified the suppressive effects of $\mathrm{H}_{2} \mathrm{~S}$ on hippocampal oxidative stress in CUMSexposed rats. We also demonstrated that $\mathrm{H}_{2} \mathrm{~S}$ significantly rescues the downregulation of BDNF expression in the hippocampus of CUMS-exposed rats. Meanwhile, K252a, a BDNF-TrkB pathway inhibitor, abolished the protective effects of $\mathrm{H}_{2} \mathrm{~S}$ against CUMS-induced oxidative stress. Taken together, we identified a critical role of $\mathrm{H}_{2} \mathrm{~S}$ in protection against CUMS-induced oxidative stress in hippocampus, as a result of upregulation of BDNF-TrkB pathway.

\section{Materials and Methods}

2.1. Animals. Adult male Sprague-Dawley (SD) rats (250$280 \mathrm{~g}$ ) were purchased from the Hunan SJA Laboratory Animal Center (Changsha, Hunan, China). Rats were housed individually and given free access to food and water under a normal $12 \mathrm{~h}$ light/dark schedule (lights on at 07:00 a.m.). Room temperature was maintained at $22 \pm 1^{\circ} \mathrm{C}$ and relative humidity of $55 \% \pm 5 \%$. Rats were allowed 7 days to acclimatize themselves to the housing conditions before the beginning of the experiments. All the procedures were strictly conducted according to the National Institutes of Health Guide for the Care and Use of Laboratory Animals and were approved by the Animal Use and Protection Committee of University of South China.

2.2. Reagents. Sodium hydrosulfide (NaHS, a donor of $\mathrm{H}_{2} \mathrm{~S}$ ) and $\mathrm{K} 252 \mathrm{a}$ (a selective pharmacological p-TrkB inhibitor) were purchased from Sigma (Sigma, St. Louis, MO, USA). Specific monoclonal antibodies for detecting BDNF and p-TrkB were obtained from Epitomic Inc. (Burlingame, UK). $\beta$-actin antibody and goat anti-rat antibody were purchased from Proteintech (Danvers, MA, USA). The 4hydroxy-2-trans-nonenal (4-HNE) assay kit, malondialdehyde (MDA) assay kit, superoxide dismutase (SOD) assay kit, and glutathione (GSH) enzyme-linked immunosorbent assay (ELISA) kits were bought from USCN Life Science Inc. (Wuhan, Hubei, China). Bicinchoninic Acid (BCA) Protein Assay Kit was obtained from Beyotime Institute of Biotechnology (Shanghai, China).

2.3. Lateral Ventricle Cannulation. Rats were first anesthetized with sodium pentobarbital (60 mg/kg, i.p., Sigma, St. Louis, MO, USA) and then placed in stereotaxic apparatus for operation; a permanent guide cannula was aseptically and stereotaxically implanted into the right lateral ventricle of rats using the coordinates (AP, $-1.0 \mathrm{~mm}$; $\mathrm{ML},+2 \mathrm{~mm}$; DV, $+3.5 \mathrm{~mm}$ ). After stereotaxic surgery, home-cage behavior and wound healing were monitored. Body weight recovery to no less than $90 \%$ of presurgery amount within 7 days was the inclusion criteria.

2.4. CUMS Procedure. Cannulated rats were exposed to a series of variable stressors per day for 2 weeks as described previously with minor modifications [25]. Briefly, animals were exposed to the series stress regime consisting of $24 \mathrm{~h}$ food deprivation, $24 \mathrm{~h}$ water deprivation, exposure to an empty bottle for $1 \mathrm{~h}, 7 \mathrm{~h}$ cage tilt $\left(45^{\circ}\right)$, overnight illumination, $24 \mathrm{~h}$ soiled cage ( $200 \mathrm{~mL}$ of water in 100 -g sawdust bedding), $30 \mathrm{~min}$ forced swimming at $8^{\circ} \mathrm{C}, 3 \mathrm{~h}$ physical restraint, and $24 \mathrm{~h}$ exposure to a unfamiliar object (e.g., a piece of plastic). All of these stressor episodes were randomly scheduled over a 1-week period and repeated throughout the 4-week experiment.

2.5. Drug Administration. CUMS rats were exposed to one stressor per day for 2 weeks and then received 2 weeks of $\mathrm{NaHS}(0.03$ or $0.1 \mathrm{mmol} / \mathrm{kg} /$ day $)$ or saline intraperitoneal injections or K252a $(1 \mu \mathrm{g})$ intracerebroventricularly (i.c.v.) during continuous CUMS treatment. Control rats were left undisturbed in their home cages with the exception of general handling for 2 weeks and intraperitoneally injected with saline in the last 2 weeks.

2.6. Novelty-Suppressed Feeding Test. The Novelty-suppressed feeding test (NSFT) was performed according to previous protocols [26], with a minor modification. Rats were initially food deprived for $24 \mathrm{~h}$ in their home cages and then transferred to a new chamber without habituation. Regular food pellets (weighed) were placed in the center of the testing room. Each subject was first placed in a corner of the testing area. The latency to begin to chew the pellet was recorded. And then rats were transferred to their home cage; the amount of food consumed for 30 min was measured.

2.7. Elevated Plus-Maze Test. Anxiolytic activity was measured in the elevated plus-maze (EPM) test [27]. In this test, the maze (EPMR; Shanghai Jiliang Software Technology Co. Ltd, Shanghai, China) consisted of four crossed narrow arms (50 cm long, $10 \mathrm{~cm}$ wide), with two open arms and two closed arms $(40 \mathrm{~cm}$ high). The apparatus was elevated $70 \mathrm{~cm}$ from the floor. During the EPM test, rats were individually first 


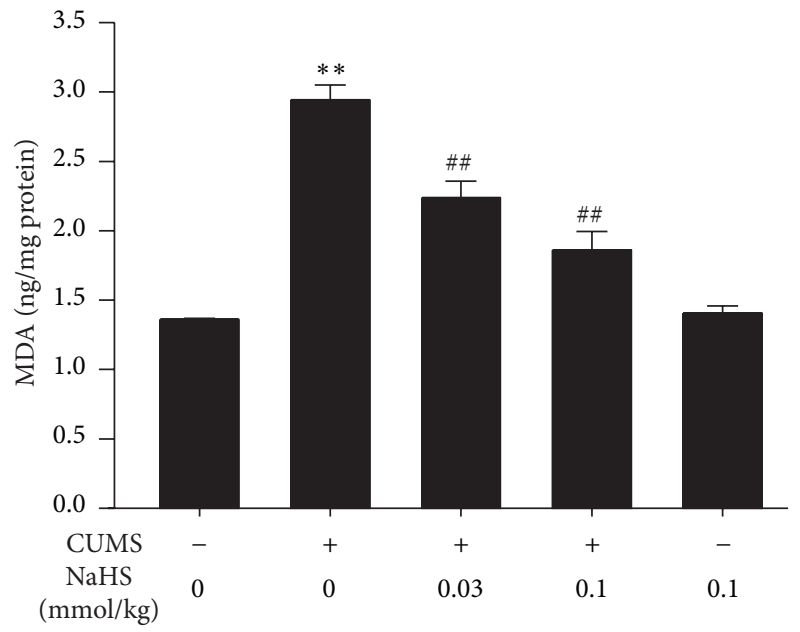

(a)

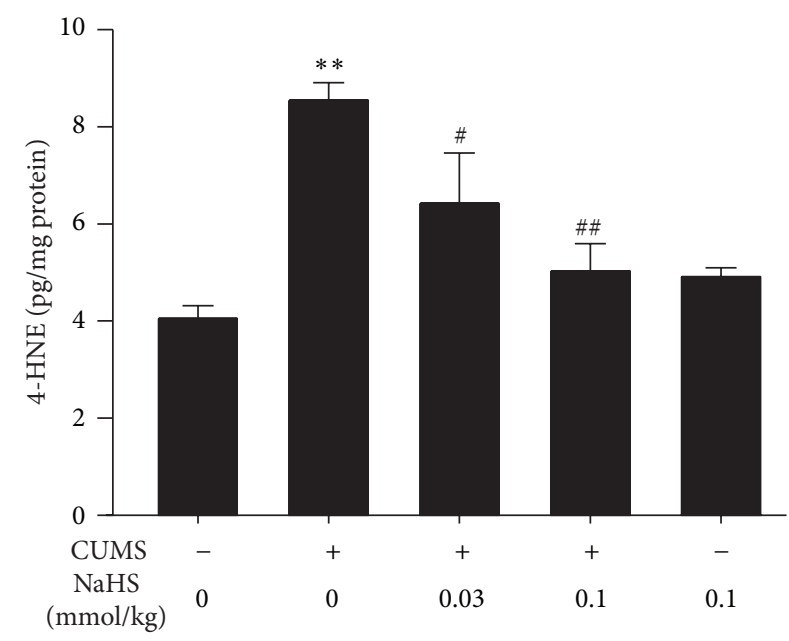

(b)

FIGURE 1: Effect of $\mathrm{H}_{2} \mathrm{~S}$ on the generations of MDA and 4-HNE in the hippocampus of CUMS-exposed rats. Rats were exposed to CUMS for $2 \mathrm{w}$ and then cotreated with NaHS ( 0.03 or $0.1 \mathrm{mmol} / \mathrm{kg} / \mathrm{day}$, i.p.) and CUMS for $2 \mathrm{w}$. The levels of MDA (a) and 4 -HNE (b) in the hippocampus of rats were detected by ELISA kit. Values are expressed as mean $\pm \operatorname{SEM}(n=3-5 /$ group $) .{ }^{* *} P<0.01$, versus the control group; ${ }^{\#} P<0.05$, ${ }^{\# \#} P<0.01$, versus the CUMS- treated group.

placed in the central zone of the EPM, head facing an open arm, and the rat were allowed to freely explore the maze for $5 \mathrm{~min}$. The total number of arm entries and the percentage of entries into and the proportion of time spent in the open arms were assessed.

2.8. Biochemical Analysis for MDA, 4-HNE, SOD, and GSH. The hippocampal tissues were homogenized $(10 \% \mathrm{w} / \mathrm{v})$ with $0.1 \mathrm{~mol} / \mathrm{L}$ of PBS and centrifuged at $12,000 \mathrm{~g}$ for $10 \mathrm{~min}$. The supernatants were collected and the total protein concentration was quantified by BCA Protein Assay. The levels of MDA, 4-HNE, and GSH and the activity of SOD in the supernatant of hippocampus were, respectively, measured by enzyme-linked immunosorbent assay (ELISA) under standard conditions, according to the manufacturer's instructions on the reagent kits. The absorbance was read at $450 \mathrm{~nm}$ using a microplate reader.

2.9. Western Blot Analysis for the Levels of BDNF and pTrkB Protein Expression. Protein from hippocampal tissue was homogenized with ice-cold homogenizing buffer and incubated on ice for $30 \mathrm{~min}$. The homogenates were centrifuged at $14,000 \mathrm{~g}$ for $10 \mathrm{~min}$ at $4^{\circ} \mathrm{C}$. The total protein of supernatant was subsequently measured by using BCA assay kit (Beyotime, Shanghai, China). Equal amounts of total protein $(25 \mu \mathrm{g} /$ lane $)$ were separated on $8-12 \%$ SDS-PAGE and electrotransferred to PVDF membranes. After blocking the membranes with TBST containing 5\% nonfat dried milk for at least $2 \mathrm{~h}$ at room temperature, membranes were incubated with the primary antibody (anti-BDNF, 1:1000, Epitomics; anti-p-TrkB, 1:1000, Epitomics; $\beta$-actin 1:2000, Proteintech) at $4^{\circ} \mathrm{C}$ overnight. Then the blots were washed three times for $8 \mathrm{~min}$ by using TBST and incubated with secondary antibody for $1 \mathrm{~h}$. After washing, protein bands were analyzed using the enhanced chemiluminescence detection system (BeyoECLPlus kit, Beyotime, P0018). The signal of the immunoblot was analyzed using Image J software and calculated with $\beta$-actin (a loading control).

2.10. Statistical Analysis. All data are expressed as mean \pm SEM. The significance of intergroup differences was evaluated by one-way analyses of variance, followed by LSD post hoc tests. A value of $P<0.05$ was considered significant.

\section{Results}

3.1. $\mathrm{H}_{2} \mathrm{~S}$ Decreases the Levels of MDA and 4-HNE in the Hippocampus of CUMS-Exposed Rats. MDA and 4-HNE are the markers of lipid peroxidation to indicate the oxidative stress level. There was a significant decrease in the levels of MDA (Figure 1(a)) and 4-HNE (Figure 1(b)) in the hippocampus of CUMS-exposed rats as compared with those of control rats. However, treatment with NaHS (a donor of $\mathrm{H}_{2} \mathrm{~S}, 0.03$ or $0.1 \mathrm{mmol} / \mathrm{kg} /$ day, i.p., for $2 \mathrm{w}$ ) significantly reduced the levels of MDA (Figure 1(a)) and 4-HNE (Figure 1(b)) in the hippocampus of CUMS-exposed rats. These data indicated that $\mathrm{H}_{2} \mathrm{~S}$ prevents CUMS-induced hippocampal oxidative stress.

3.2. $\mathrm{H}_{2} \mathrm{~S}$ Increases the SOD Activity and GSH Level in the Hippocampus of CUMS-Exposed Rats. We also investigated the effects of $\mathrm{H}_{2} \mathrm{~S}$ on the SOD activity and GSH level in the hippocampus of CUMS-treated rats. The SOD activity (Figure 2(a)) and GSH level (Figure 2(b)) were significantly reduced in the hippocampus of CUMS-treated rats. However, treatment with $\mathrm{NaHS}(0.03$ or $0.1 \mathrm{mmol} / \mathrm{kg} /$ day, i.p., for $2 \mathrm{w})$ increased the SOD activity and GSH level in the hippocampus of CUMS-treated rats, which further indicated the protective 


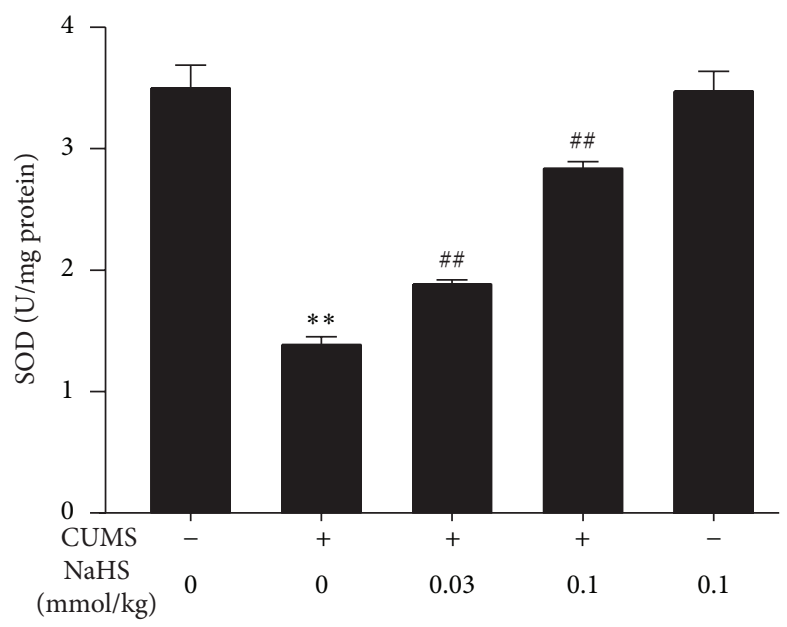

(a)

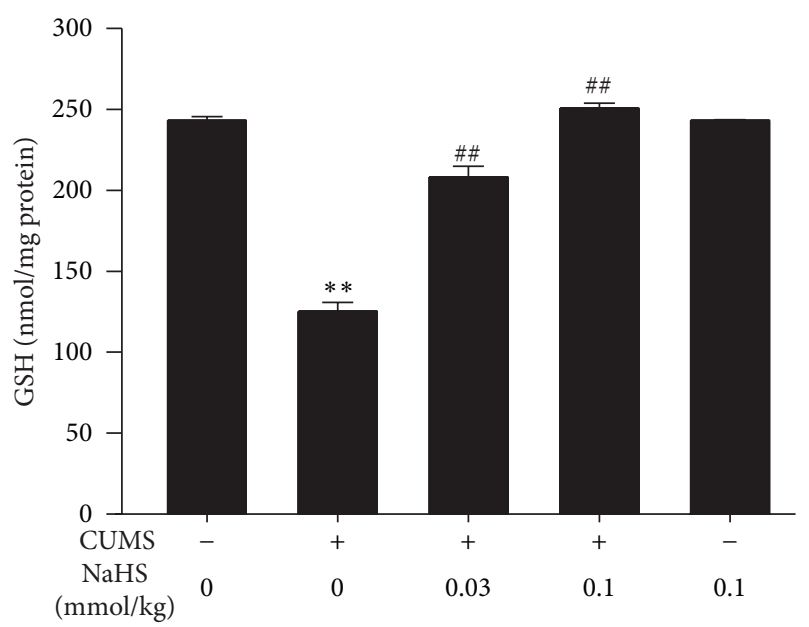

(b)

FIGURE 2: Effect of $\mathrm{H}_{2} \mathrm{~S}$ on the SOD activity and GSH level in the hippocampus of CUMS-exposed rats. Rats were exposed to CUMS for $2 \mathrm{w}$ and then cotreated with NaHS ( 0.03 or $0.1 \mathrm{mmol} / \mathrm{kg} /$ day, i.p.) and CUMS for $2 \mathrm{w}$. The SOD activity (a) and GSH level (b) in the hippocampus of rats were detected by ELISA kit. Values are expressed as mean $\pm \operatorname{SEM}(n=3-5 /$ group $) .{ }^{* *} P<0.01$, versus the control group, ${ }^{\# \#} P<0.01$, versus the CUMS-treated alone group.

action of $\mathrm{H}_{2} \mathrm{~S}$ against CUMS-exerted hippocampal oxidative stress.

\section{3. $\mathrm{H}_{2} \mathrm{~S}$ Upregulates the Level of BDNF and p-TrkB Protein} in the Hippocampus of CUMS-Exposed Rats. To determine whether BDNF/TrkB pathway is involved in the protective effect of $\mathrm{H}_{2} \mathrm{~S}$ against CUMS-induced hippocampal oxidative stress, we first explored the effects of $\mathrm{H}_{2} \mathrm{~S}$ on the expression of BDNF and $\mathrm{p}$-TrkB protein in CUMS-exposed rats. After $4 \mathrm{w}$ exposure of CUMS, the expressions of BDNF (Figure 3(a)) and $\mathrm{p}$-TrkB (Figure 3(b)) protein in the hippocampus were significantly decreased in the hippocampus of rats. However, after treatment with $\mathrm{NaHS}(0.03$ or $0.1 \mathrm{mmol} / \mathrm{kg} /$ day, i.p., for $2 \mathrm{w}$ ), the expressions of BDNF (Figure 3(a)) and p$\operatorname{TrkB}$ (Figure 3(b)) proteins in the hippocampus of CUMSexposed rats were markedly increased, which indicated that $\mathrm{H}_{2} \mathrm{~S}$ upregulates the hippocampal BDNF-TrkB pathway in CUMS-exposed rats.

3.4. K252a Reverses the Inhibitory Role of $\mathrm{H}_{2} \mathrm{~S}$ in CUMSIncreased MDA and 4-HNE in the Hippocampus. To confirm the mediatory role of BDNF-TrkB pathway in the protection of $\mathrm{H}_{2} \mathrm{~S}$ against CUMS-induced hippocampal oxidative stress, we further explored whether K252a, a specific BDNF-TrkB pathway inhibitor, reverses the protective role of $\mathrm{H}_{2} \mathrm{~S}$ against hippocampal oxidative stress in CUMS-exposed rats. As shown in Figure 4, K252a (1 $\mu$ g, i.c.v.) eliminated the protection of $\mathrm{NaHS}(0.1 \mathrm{mmol} / \mathrm{kg}$, i.p. $)$ against CUMSinduced increases in hippocampal MDA (Figure 4(a)) and 4-HNE (Figure 4(b)) levels, while treatment with K252a $(1 \mu \mathrm{g})$ alone did not affect the MDA (Figure 4(a)) and 4HNE (Figure 4(b)) levels in control rats, which indicated that $\mathrm{K} 252 \mathrm{a}$ blocks the protection of $\mathrm{H}_{2} \mathrm{~S}$ against hippocampal oxidative stress in CUMS-exposed rats.
3.5. K252a Reverses the Upregulatory Role of $\mathrm{H}_{2} \mathrm{~S}$ in the SOD Activity and GSH Level in the Hippocampus of CUMS-Exposed Rats. To further determine the mediatory role of BDNF-TrkB pathway in the protection of $\mathrm{H}_{2} \mathrm{~S}$ against CUMS-induced hippocampal oxidative stress, we also explored whether K252a blocks the upregulatory role of $\mathrm{H}_{2} \mathrm{~S}$ in the SOD activity and GSH level. K252a (1 $\mu$ g, i.c.v.) significantly decreased the SOD activity (Figure 5(a)) and GSH level (Figure 5(b)) in the hippocampus of NaHS $(0.1 \mathrm{mmol} / \mathrm{kg}$, i.p.) and CUMS cotreated rats. This result also suggests that BDNF/TrkB pathway is involved in the protection of $\mathrm{H}_{2} \mathrm{~S}$ against CUMSinduced hippocampal oxidative stress.

3.6. $\mathrm{H}_{2} \mathrm{~S}$ Exerts Antidepressive- and Anxiolytic-Like Effects on CUMS-Exposed Rats. We further verified the ability of $\mathrm{H}_{2} \mathrm{~S}$ to prevent CUMS-induced depression- and anxiety-like behaviors. The NSF test has been suggested to have high predictive validity for antianxiety activity [26]. As shown in Figure 6(a), CUMS-exposed rats displayed depressionlike behaviors reflected by an increase in the time of latency to feed; however, treatment with NaHS (0.03 or $0.1 \mathrm{mmol} / \mathrm{kg} / \mathrm{day}$, i.p., for $2 \mathrm{w}$ ) decreases the latency to feed in the CUMS-exposed rats, reflecting the antidepressivelike action of $\mathrm{H}_{2} \mathrm{~S}$. NaHS treatment did not affect the food consumption at home cage during the 30 min test session (Figure 6(b)), indicating that the antidepressive-like effect of $\mathrm{NaHS}$ was not due to the possibility that NaHS affected the normal appetite and feeding. To verify the possibility that $\mathrm{H}_{2} \mathrm{~S}$ exerts an anxiolytic-like activity in CUMS rats, rats were subjected to the EPM test. After they were treated with NaHS ( 0.03 or $0.1 \mathrm{mmol} / \mathrm{kg} / \mathrm{day}$, intraperitoneally) for $2 \mathrm{w}$, we found an increase in the proportion of entries into (Figure 3(c)) and the time spent in (Figure 3(d)) the open arms, without significant change in total arm entries (Figure 3(e)) compared 

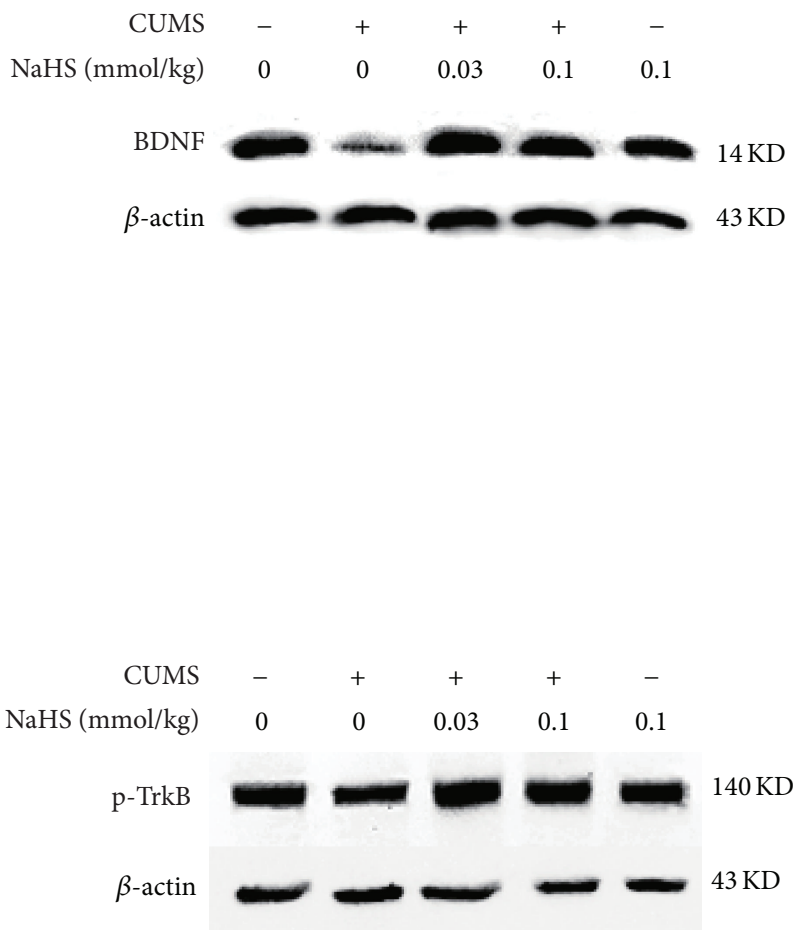

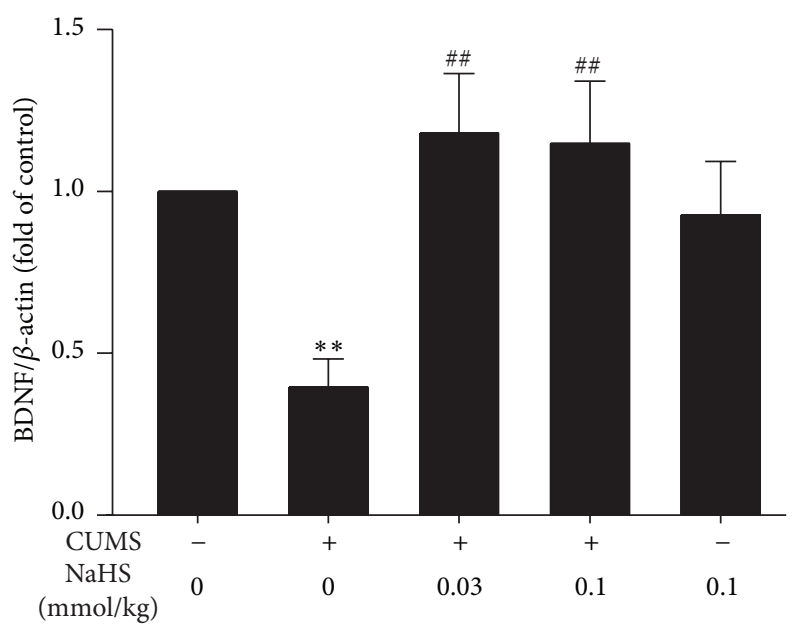

(a)

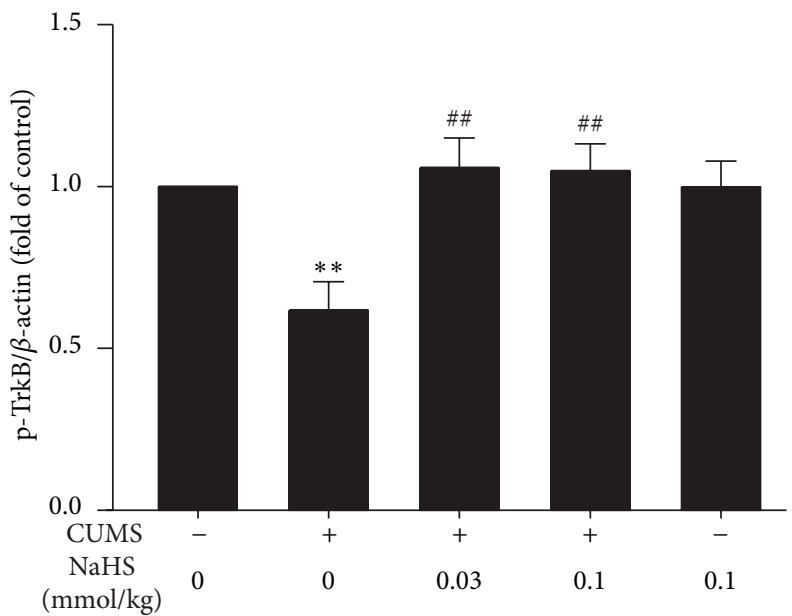

(b)

FIGURE 3: Effect of $\mathrm{H}_{2} \mathrm{~S}$ on the expressions of BDNF and p-TrkB protein in the hippocampus of CUMS-treated rat. Rats were exposed to CUMS for $2 \mathrm{w}$ and then cotreated with NaHS $(0.03$ or $0.1 \mathrm{mmol} / \mathrm{kg} /$ day, i.p.) and CUMS for $2 \mathrm{w}$. Immunoblot was used to analyze and quantify the expressions of BDNF and p-TrkB in the hippocampus of rats, and $\beta$-actin was used as an internal control. Values were expressed as the mean \pm SEM of three independent experiments. ${ }^{* *} P<0.01$, versus control group; ${ }^{\# \#} P<0.01$, versus CUMS-treated along group.

to the saline-treated CUMS rats, indicating an anxiolytic-like effect of $\mathrm{H}_{2} \mathrm{~S}$ in CUMS rats.

\section{Discussion}

$\mathrm{H}_{2} \mathrm{~S}$, a novel endogenous gaseous mediator, has been recognized as an antioxidant $[28,29]$ and our group has demonstrated the decrease of endogenous $\mathrm{H}_{2} \mathrm{~S}$ in the hippocampus of CUMS-exposed rats [18]. Emerging evidence supports that brain-derived neurotrophic factor (BDNF) has neuroprotective effect. In the present work, we investigated whether $\mathrm{H}_{2} \mathrm{~S}$ inhibits CUMS-induced hippocampal oxidative stress and whether BDNF/TrkB pathway mediates this inhibitory role of $\mathrm{H}_{2} \mathrm{~S}$. We found that $\mathrm{H}_{2} \mathrm{~S}$ decreased oxidative stress and increased the expressions of BDNF and p-TrkB in the hippocampus of CUMS-exposed rats. Moreover, this protection effect of $\mathrm{H}_{2} \mathrm{~S}$ against oxidative stress was abolished by $\mathrm{K} 252 \mathrm{a}$, an inhibitor of $\operatorname{TrkB}$. This is the first report demonstrating the protection of $\mathrm{H}_{2} \mathrm{~S}$ against CUMS-induced hippocampal oxidative stress, as a result of upregulation of BDNF-TrkB pathway.

Oxidative stress contributes to the pathophysiologic cascade relating with hippocampus injury [30]. Similarly, cumulative evidence indicates that oxidative stress in the hippocampus exerts a vital role in CUMS-induced pathogenesis of rodents [31-37]. Lowered antioxidant enzyme activity within the central nervous system may impair protection against reactive oxygen species (ROS), leading to damage to intracellular structure such as DNA, fatty acid, and protein [38-41]. Recently, the potential antioxidant and cytoprotective effects of $\mathrm{H}_{2} \mathrm{~S}$ have been a focus of research [42]. It has shown that $\mathrm{H}_{2} \mathrm{~S}$ scavenges oxidants and reactive aldehydes, such as 4-HNE, MDA, and peroxynitrite, by modulating the GSH content and SOD activity [43-46]. Thus, we investigated whether $\mathrm{H}_{2} \mathrm{~S}$ protects hippocampus against CUMS-induced oxidative stress. In our study, CUMS-treated 


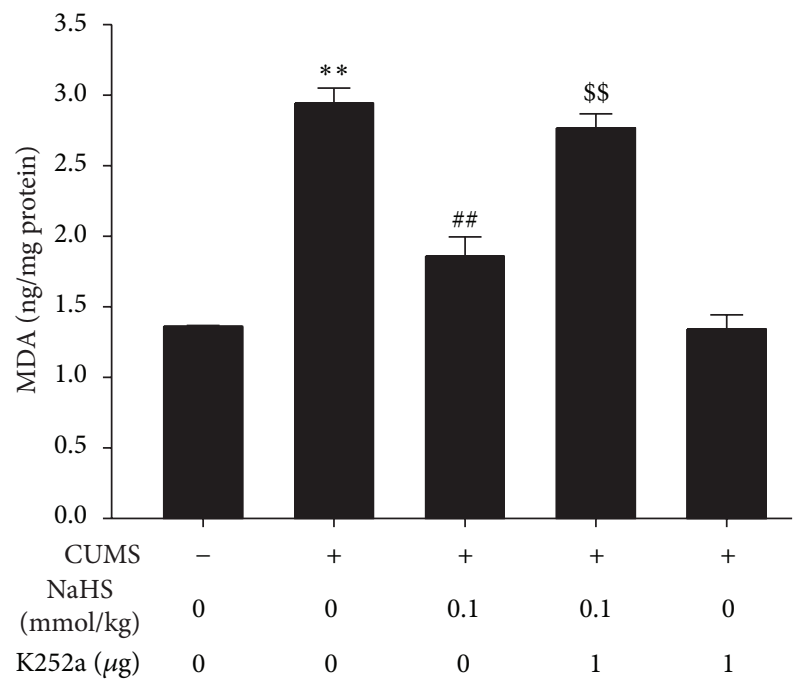

(a)

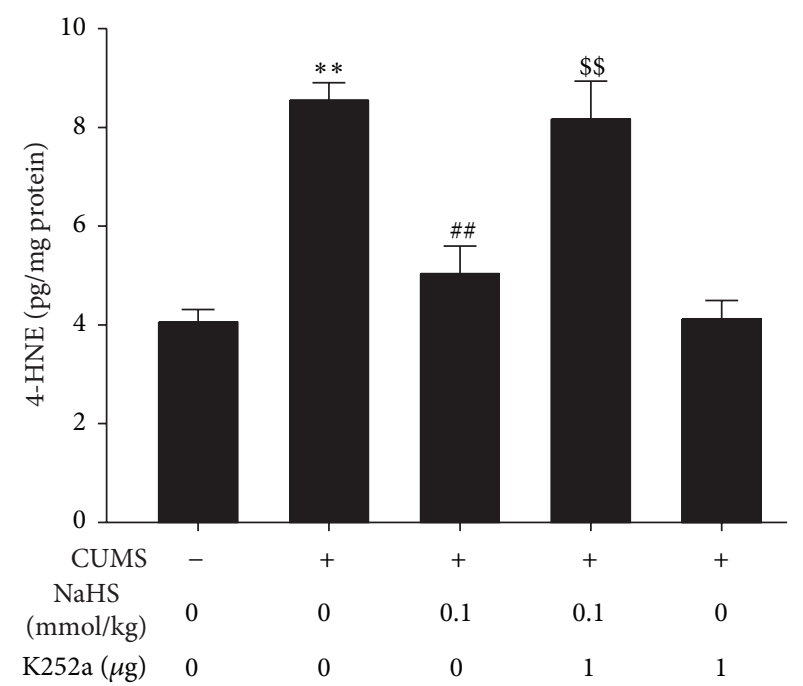

(b)

Figure 4: Effect of k252a on $\mathrm{H}_{2} \mathrm{~S}$-caused decrease in MDA and 4-HNE generation in the hippocampus of CUMS rats. The levels of MDA (a) and 4 -HNE (b) in the hippocampus of rats were detected by ELISA kit. Values are expressed as mean \pm SEM $\left(n=5-6 /\right.$ group). ${ }^{* *} P<0.01$, versus control group; ${ }^{\# \#} P<0.01$, versus CUMS-treated alone group; ${ }^{\$ \$} P<0.01$, versus CUMS and $\mathrm{H}_{2} \mathrm{~S}$-treated group.

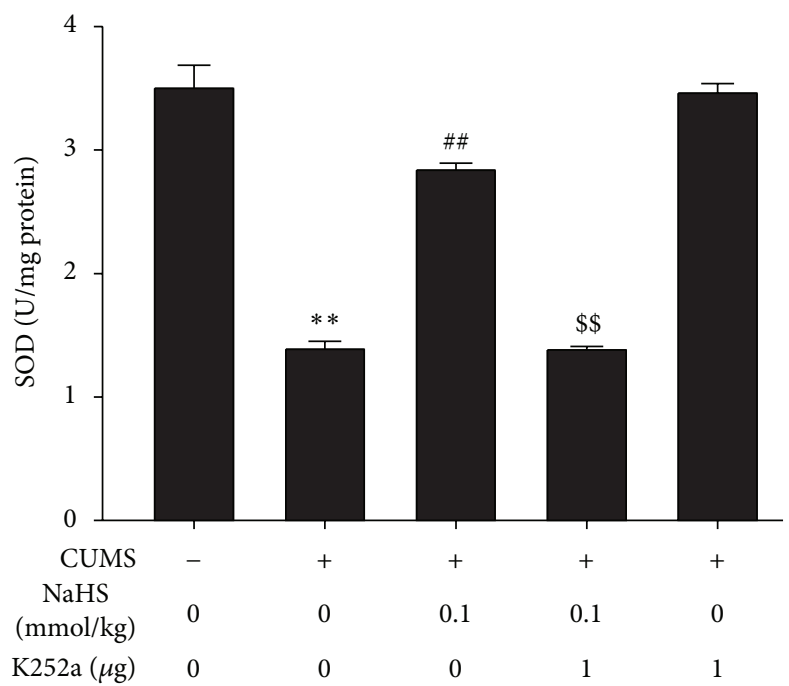

(a)



(b)

FIGURE 5: Effect of k252a on $\mathrm{H}_{2} \mathrm{~S}$ upregulated SOD activity and GSH level in the hippocampus of CUMS-exposed rats. The SOD activity (a) and GSH level (b) in the hippocampus of rats were detected by ELISA kit. Values are expressed as mean $\pm \operatorname{SEM}(n=5-6 /$ group $) .{ }^{* *} P<0.01$, versus control group; ${ }^{\# \#} P<0.01$, versus CUMS-treated alone group; ${ }^{\$ \$} P<0.01$, versus CUMS and $\mathrm{H}_{2} \mathrm{~S}$-treated group.

rats exerted a significant increase in the concentration of lipid peroxidation, MDA, and 4-HNE and marked reduction in the GSH level and SOD activity of the hippocampus. However, these parameters were significantly reversed by NaHS (a donor of $\mathrm{H}_{2} \mathrm{~S}$ ) administration. NaHS notably decreased the levels of MDA and 4-HNE and increased the GSH level and SOD activity in the hippocampus of CUMS-treated rats. These data suggested that $\mathrm{H}_{2} \mathrm{~S}$ attenuates oxidative stress in the hippocampus of CUMS-exposed rats. Considering that CUMS is well established to cause depressive- and anxietylike behaviors, it would be important to verify the ability of
$\mathrm{H}_{2} \mathrm{~S}$ to abolish CUMS-induced depressive- and anxiety-like behaviors. In the present work, we found that $\mathrm{H}_{2} \mathrm{~S}$ prevented CUMS-caused depressive-like behavior in the NSF test and anxiety-like behavior in the EPM test, which are consistent with our previous study [47].

BDNF modulates cellular function by activation of its specific tyrosine kinase receptor, TrkB. Numerous studies have also documented that BDNF has neuroprotective effects [48-51]. Interestingly, our previous study confirmed that $\mathrm{H}_{2} \mathrm{~S}$ increases the expression of BDNF protein in the hippocampus of rats [52]. BDNF also possesses antioxidant 




(a)

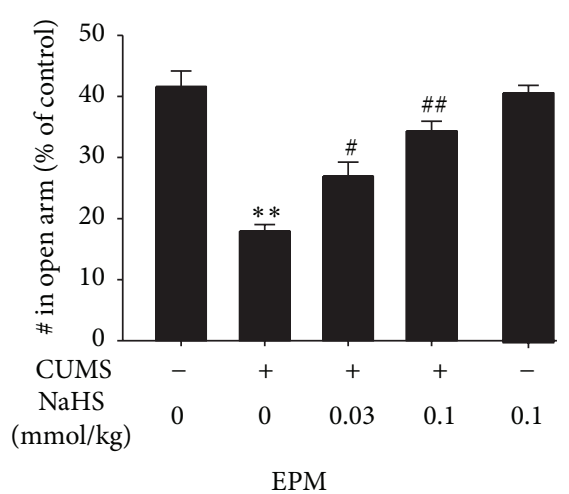

(c)

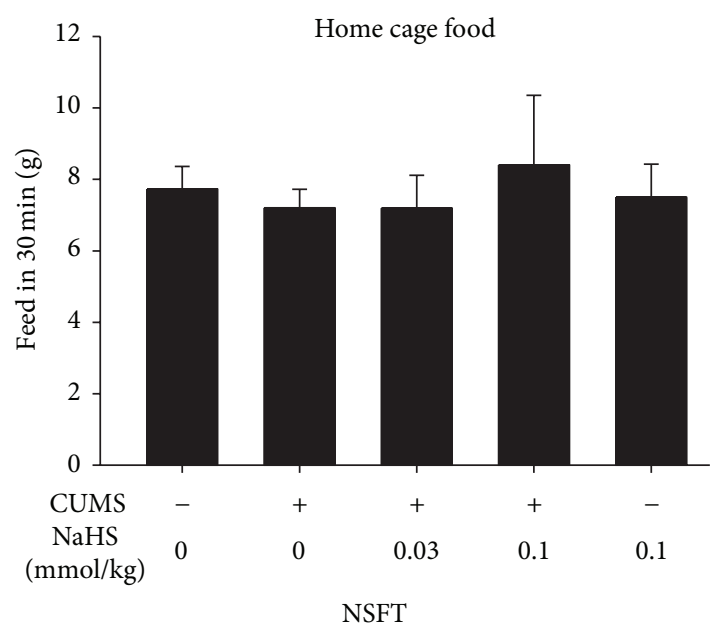

(b)



(d)

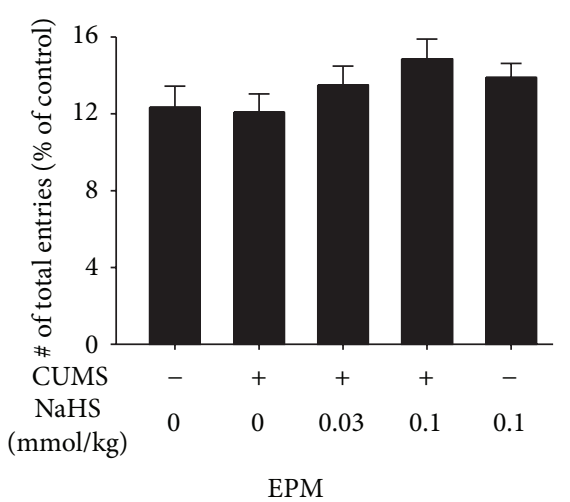

(e)

FIgURE 6: Effect of $\mathrm{H}_{2} \mathrm{~S}$ on depressive- and anxiety-like behaviors in CUMS-exposed rats. Rats were exposed to CUMS for $2 \mathrm{w}$ and then cotreated with NaHS (0.03 or $0.1 \mathrm{mmol} / \mathrm{kg} / \mathrm{day}$, i.p.) and CUMS for $2 \mathrm{w}$. (a)-(b) The depressive-like behavior was evaluated using noveltysuppressed feeding test (NSFT). (c)-(e) The anxiety-like behavior was evaluated using elevated plus-maze $\left(\right.$ EPM) test. ${ }^{* *} P<0.01$ versus control group, ${ }^{\# \#} P<0.01$, versus CUMS-treated alone group. ${ }^{\# \# \#} P<0.001$, versus CUMS-treated group. Data represent group mean \pm SEM ( $n=7-10$ group).

activities [53]. Meanwhile, our data are consistent with previously reported data that CUMS decreases BDNF levels in the hippocampus [34]. We also found that treatment of rat with NaHS intraperitoneal injections increased the expression of BDNF and $\mathrm{p}$-TrkB proteins in the hippocampus of CUMS-exposed rats. Therefore, we speculate whether the BDNF-TrkB pathway mediates the protection of $\mathrm{H}_{2} \mathrm{~S}$ against oxidative stress in the hippocampus of CUMS-treated rats. We infused K252a, the TrkB receptor antagonist, to inhibit BDNF-TrkB signaling in the brain. Subsequently, we detected the two endogenous aldehydes levels of MDA and HNE, which are commonly used as a marker of oxidative stress $[54,55]$. We found that $\mathrm{K} 252 \mathrm{a}$ blunt $\mathrm{H}_{2} \mathrm{~S}$ antioxidant effect in CUMS-exposed rats. In line with this, the inhibitory role of $\mathrm{H}_{2} \mathrm{~S}$ in CUMS-increased MDA and 4-HNE was reversed by K252a in the hippocampus of CUMS-treated rats. In addition, K252a was able to completely block the upregulatory role of
$\mathrm{H}_{2} \mathrm{~S}$ in the SOD activity and GSH level in the hippocampus of CUMS-exposed rats. Collectively, these findings suggest that $\mathrm{BDNF} / \mathrm{TrkB}$ pathway mediates the protection of $\mathrm{H}_{2} \mathrm{~S}$ against CUMS-induced hippocampal oxidative stress.

Finally, our data suggest that $\mathrm{H}_{2} \mathrm{~S}$ protects against CUMS-induced hippocampal oxidative stress by modulating $\mathrm{BDNF} / \mathrm{TrkB}$ singling. It is still unclear whether $\mathrm{H}_{2} \mathrm{~S}$-derived bioactive molecules will be involved in regulating hippocampal oxidative system. Interestingly, recent study shows that endogenous diffusible polysulfides $\left(\mathrm{H}_{2} \mathrm{~S}_{n}\right)$ in rat brain are released from $\mathrm{H}_{2} \mathrm{~S}[56,57]$. Further experimental and clinical studies are still needed to determine the antioxidant activity of $\mathrm{H}_{2} \mathrm{~S}$.

In conclusion, the present study demonstrated that $\mathrm{H}_{2} \mathrm{~S}$ inhibits CUMS-induced hippocampal oxidative stress and upregulates the hippocampal BDNF-TrkB pathway in CUMS-exposed rats and that the blockage of BDNF-TrkB 
pathway reverses the protection of $\mathrm{H}_{2} \mathrm{~S}$ against CUMSinduced oxidative stress. Our data establish a key role of $\mathrm{H}_{2} \mathrm{~S}$ against CUMS-induced hippocampal oxidative stress through BDNF-TrkB pathway and provide a basis for investigating $\mathrm{H}_{2} \mathrm{~S}$ as a therapeutic approach for the pathogenesis of stress.

\section{Competing Interests}

The authors declare that they have no competing interests.

\section{Authors' Contributions}

Min $\mathrm{Hu}$ and Wei Zou contributed equally to this work.

\section{Acknowledgments}

This study was supported by Natural Science Foundation of China (81371485), Zhengxiang Scholar Program of University of South China (2014-004), and the Construct Program of the Key Discipline in Hunan Province.

\section{References}

[1] B. S. McEwen, "Protective and damaging effects of stress mediators: central role of the brain," Progress in Brain Research, vol. 122, pp. 25-34, 2000.

[2] D. S. Charney and H. K. Manji, "Life stress, genes, and depression: multiple pathways lead to increased risk and new opportunities for intervention," Science's STKE, vol. 2004, no. 225, p. re5, 2004.

[3] J. D. Bremner, "Does stress damage the brain," Biological Psychiatry, vol. 45, no. 7, pp. 797-805, 1999.

[4] R. S. Duman and L. M. Monteggia, "A neurotrophic model for stress-related mood disorders," Biological Psychiatry, vol. 59, no. 12, pp. 1116-1127, 2006.

[5] A. L. Lee, W. O. Ogle, and R. M. Sapolsky, "Stress and depression: possible links to neuron death in the hippocampus," Bipolar Disorders, vol. 4, no. 2, pp. 117-128, 2002.

[6] M. Moretti, A. Colla, G. de Oliveira Balen et al., "Ascorbic acid treatment, similarly to fluoxetine, reverses depressivelike behavior and brain oxidative damage induced by chronic unpredictable stress," Journal of Psychiatric Research, vol. 46, no. 3, pp. 331-340, 2012.

[7] M. F. Schaalan and N. N. Nassar, "Effects of octreotide in chronically mild stressed rats: possible role of immune and oxidative stress pathways," Neurochemical Research, vol. 36, no. 10, pp. 1717-1723, 2011.

[8] E. Lowicka and J. Beltowski, "Hydrogen sulfide (H2S)-the third gas of interest for pharmacologists," Pharmacological Reports, vol. 59, no. 1, pp. 4-24, 2007.

[9] R. Wang, "Hydrogen sulfide: The third gasotransmitter in biology and medicine," Antioxidants and Redox Signaling, vol. 12, no. 9, pp. 1061-1064, 2010.

[10] R. Wang, "Two's company, three's a crowd: can $\mathrm{H}_{2} \mathrm{~S}$ be the third endogenous gaseous transmitter?" The FASEB Journal, vol. 16, no. 13, pp. 1792-1798, 2002.

[11] K. Abe and H. Kimura, "The possible role of hydrogen sulfide as an endogenous neuromodulator," The Journal of Neuroscience, vol. 16, no. 3, pp. 1066-1071, 1996.
[12] H. Kimura, "Hydrogen sulfide induces cyclic AMP and modulates the NMDA receptor," Biochemical and Biophysical Research Communications, vol. 267, no. 1, pp. 129-133, 2000.

[13] Y. Nagai, M. Tsugane, J.-I. Oka, and H. Kimura, "Hydrogen sulfide induces calcium waves in astrocytes," The FASEB Journal, vol. 18, no. 3, pp. 557-559, 2004.

[14] S. W. Lee, Y.-S. Hu, L.-F. Hu et al., "Hydrogen sulphide regulates calcium homeostasis in microglial cells," Glia, vol. 54, no. 2, pp. 116-124, 2006.

[15] Y. Kimura, Y.-I. Goto, and H. Kimura, "Hydrogen sulfide increases glutathione production and suppresses oxidative stress in mitochondria," Antioxidants and Redox Signaling, vol. 12, no. 1, pp. 1-13, 2010.

[16] Y. Kimura and H. Kimura, "Hydrogen sulfide protects neurons from oxidative stress," The FASEB Journal, vol. 18, no. 10, pp. 1165-1167, 2004.

[17] L.-H. Jiang, X. Luo, W. A. He, X.-X. Huang, and T.-T. Cheng, "Effects of exogenous hydrogen sulfide on apoptosis proteins and oxidative stress in the hippocampus of rats undergoing heroin withdrawal," Archives of Pharmacal Research, vol. 34, no. 12, pp. 2155-2162, 2011.

[18] H. Tan, W. Zou, J. Jiang et al., "Disturbance of hippocampal $\mathrm{H}_{2} \mathrm{~S}$ generation contributes to CUMS-induced depressionlike behavior: involvement in endoplasmic reticulum stress of hippocampus," Acta Biochimica et Biophysica Sinica, vol. 47, no. 4, pp. 285-291, 2015.

[19] D. K. Binder and H. E. Scharfman, "Brain-derived neurotrophic factor," Growth Factors, vol. 22, no. 3, pp. 123-131, 2004.

[20] Q. Yan, M. J. Radeke, C. R. Matheson, J. Talvenheimo, A. A. Welcher, and S. C. Feinstein, "Immunocytochemical localization of TrkB in the central nervous system of the adult rat," Journal of Comparative Neurology, vol. 378, no. 1, pp. 135-157, 1997.

[21] M. A. Smith, S. Makino, R. Kvetnansky, and R. M. Post, "Stress and glucocorticoids affect the expression of brain-derived neurotrophic factor and neurotrophin-3 mRNAs in the hippocampus," The Journal of Neuroscience, vol. 15, no. 3, part 1, pp. 1768-1777, 1995.

[22] L.-T. Yi, J. Li, B.-B. Liu, L. Luo, Q. Liu, and D. Geng, "BDNFERK-CREB signalling mediates the role of miR-132 in the regulation of the effects of oleanolic acid in male mice," Journal of Psychiatry and Neuroscience, vol. 39, no. 5, pp. 348-359, 2014.

[23] N. I. Boyadjieva and D. K. Sarkar, "Cyclic adenosine monophosphate and brain-derived neurotrophic factor decreased oxidative stress and apoptosis in developing hypothalamic neuronal cells: role of microglia," Alcoholism: Clinical and Experimental Research, vol. 37, no. 8, pp. 1370-1379, 2013.

[24] J.-M. Jiang, C.-F. Zhou, S.-L. Gao et al., "BDNF-TrkB pathway mediates neuroprotection of hydrogen sulfide against formaldehyde-induced toxicity to PC12 cells," PLoS ONE, vol. 10, no. 3, Article ID e0119478, 2015.

[25] Q.-Q. Mao, S.-P. Ip, K.-M. Ko, S.-H. Tsai, and C.-T. Che, "Peony glycosides produce antidepressant-like action in mice exposed to chronic unpredictable mild stress: effects on hypothalamicpituitary-adrenal function and brain-derived neurotrophic factor," Progress in Neuro-Psychopharmacology and Biological Psychiatry, vol. 33, no. 7, pp. 1211-1216, 2009.

[26] S. R. Bodnoff, B. Suranyi-Cadotte, D. H. Aitken, R. Quirion, and M. J. Meaney, "The effects of chronic antidepressant treatment in an animal model of anxiety," Psychopharmacology, vol. 95, no. 3, pp. 298-302, 1988. 
[27] Z. Rogóz and G. Skuza, "Anxiolytic-like effects of olanzapine, risperidone and fluoxetine in the elevated plus-maze test in rats," Pharmacological Reports, vol. 63, no. 6, pp. 1547-1552, 2011.

[28] S. Taniguchi, L. Kang, T. Kimura, and I. Niki, "Hydrogen sulphide protects mouse pancreatic $\beta$-cells from cell death induced by oxidative stress, but not by endoplasmic reticulum stress," British Journal of Pharmacology, vol. 162, no. 5, pp. 11711178,2011

[29] Z.-S. Xu, X.-Y. Wang, D.-M. Xiao et al., "Hydrogen sulfide protects MC3T3-E1 osteoblastic cells against $\mathrm{H}_{2} \mathrm{O}_{2}$-induced oxidative damage-implications for the treatment of osteoporosis," Free Radical Biology and Medicine, vol. 50, no. 10, pp. 13141323, 2011.

[30] A. Ahmad, N. Rasheed, N. Banu, and G. Palit, "Alterations in monoamine levels and oxidative systems in frontal cortex, striatum, and hippocampus of the rat brain during chronic unpredictable stress," Stress, vol. 13, no. 4, pp. 355-364, 2010.

[31] H. F. Sakr, A. M. Abbas, A. Z. Elsamanoudy, and F. M. Ghoneim, "Effect of fluoxetine and resveratrol on testicular functions and oxidative stress in a rat model of chronic mild stress-induced depression," Journal of Physiology and Pharmacology, vol. 66, no. 4, pp. 515-527, 2015.

[32] R. Li, X. Wang, T. Qin, R. Qu, and S. Ma, "Apigenin ameliorates chronic mild stress-induced depressive behavior by inhibiting interleukin-1 $\beta$ production and NLRP3 inflammasome activation in the rat brain," Behavioural Brain Research, vol. 296, pp. 318-325, 2016.

[33] A. Jindal, R. Mahesh, and S. Bhatt, "Etazolate, a phosphodiesterase 4 inhibitor reverses chronic unpredictable mild stressinduced depression-like behavior and brain oxidative damage," Pharmacology Biochemistry and Behavior, vol. 105, pp. 63-70, 2013.

[34] C. B. Filho, C. R. Jesse, F. Donato et al., "Chronic unpredictable mild stress decreases BDNF and NGF levels and $\mathrm{Na}^{+}, \mathrm{K}^{+}$ATPase activity in the hippocampus and prefrontal cortex of mice: antidepressant effect of chrysin," Neuroscience, vol. 289, pp. 367-380, 2015.

[35] S. Bhatt, M. Radhakrishnan, A. Jindal, T. Devadoss, and A. K. Dhar, "Neuropharmacological evaluation of a novel 5-HT3 receptor antagonist (6g) on chronic unpredictable mild stressinduced changes in behavioural and brain oxidative stress parameters in mice," Indian Journal of Pharmacology, vol. 46, no. 2, pp. 191-196, 2014.

[36] G. Biala, K. Pekala, A. Boguszewska-Czubara, A. Michalak, M. Kruk-Slomka, and B. Budzynska, "Behavioral and biochemical interaction between nicotine and chronic unpredictable mild stress in mice," Molecular Neurobiology, 2016.

[37] S. Umukoro, O. M. Aluko, A. T. Eduviere, and O. Owoeye, "Evaluation of adaptogenic-like property of methyl jasmonate in mice exposed to unpredictable chronic mild stress," Brain Research Bulletin, vol. 121, pp. 105-114, 2016.

[38] S. D. Khanzode, G. N. Dakhale, S. S. Khanzode, A. Saoji, and R. Palasodkar, "Oxidative damage and major depression: the potential antioxidant action of selective serotonin-re-uptake inhibitors," Redox Report, vol. 8, no. 6, pp. 365-370, 2003.

[39] İ. Eren, M. Nazıroğlu, A. Demirdaş et al., "Venlafaxine modulates depression-induced oxidative stress in brain and medulla of rat," Neurochemical Research, vol. 32, no. 3, pp. 497-505, 2007.

[40] M. Maes, P. Galecki, Y. S. Chang, and M. Berk, "A review on the oxidative and nitrosative stress (O\&NS) pathways in major depression and their possible contribution to the (neuro)degenerative processes in that illness," Progress in
Neuro-Psychopharmacology and Biological Psychiatry, vol. 35, no. 3, pp. 676-692, 2011.

[41] M. Bilici, H. Efe, M. A. Köroğlu, H. A. Uydu, M. Bekaroğlu, and O. Değer, "Antioxidative enzyme activities and lipid peroxidation in major depression: Alterations by antidepressant treatments," Journal of Affective Disorders, vol. 64, no. 1, pp. 4351, 2001.

[42] J. W. Calvert, W. A. Coetzee, and D. J. Lefer, "Novel insights into hydrogen sulfide-mediated cytoprotection," Antioxidants and Redox Signaling, vol. 12, no. 10, pp. 1203-1217, 2010.

[43] J. Yin, C. Tu, J. Zhao et al., "Exogenous hydrogen sulfide protects against global cerebral ischemia/reperfusion injury via its antioxidative, anti-inflammatory and anti-apoptotic effects in rats," Brain Research, vol. 1491, pp. 188-196, 2013.

[44] S. M. Schreier, M. K. Muellner, H. Steinkellner et al., "Hydrogen sulfide scavenges the cytotoxic lipid oxidation product 4-HNE," Neurotoxicity Research, vol. 17, no. 3, pp. 249-256, 2010.

[45] M. Whiteman, N. S. Cheung, Y.-Z. Zhu et al., "Hydrogen sulphide: a novel inhibitor of hypochlorous acid-mediated oxidative damage in the brain?" Biochemical and Biophysical Research Communications, vol. 326, no. 4, pp. 794-798, 2005.

[46] M. Whiteman, J. S. Armstrong, S. H. Chu et al., "The novel neuromodulator hydrogen sulfide: an endogenous peroxynitrite 'scavenger'?" Journal of Neurochemistry, vol. 90, no. 3, pp. 765-768, 2004.

[47] W.-L. Chen, B. Xie, C. Zhang et al., "Antidepressant-like and anxiolytic-like effects of hydrogen sulfide in behavioral models of depression and anxiety," Behavioural Pharmacology, vol. 24, no. 7, pp. 590-597, 2013.

[48] E. C. Lauterbach, "Neuroprotective effects of psychotropic drugs in huntington's disease," International Journal of Molecular Sciences, vol. 14, no. 11, pp. 22558-22603, 2013.

[49] A. Chen, L. J. Xiong, Y. Tong, and M. Mao, "The neuroprotective roles of BDNF in hypoxic ischemic brain injury," Bioscience Reports, vol. 1, no. 2, pp. 167-176, 2013.

[50] R. D. Almeida, B. J. Manadas, C. V. Melo et al., "Neuroprotection by BDNF against glutamate-induced apoptotic cell death is mediated by ERK and PI3-kinase pathways," Cell Death and Differentiation, vol. 12, no. 10, pp. 1329-1343, 2005.

[51] H. Jourdi, L. Hamo, T. Oka, A. Seegan, and M. Baudry, "BDNF mediates the neuroprotective effects of positive AMPA receptor modulators against $\mathrm{MPP}+$-induced toxicity in cultured hippocampal and mesencephalic slices," Neuropharmacology, vol. 56, no. 5, pp. 876-885, 2009.

[52] H.-J. Wei, J.-H. Xu, M.-H. Li et al., "Hydrogen sulfide inhibits homocysteine-induced endoplasmic reticulum stress and neuronal apoptosis in rat hippocampus via upregulation of the BDNF-TrkB pathway," Acta Pharmacologica Sinica, vol. 35, no. 6, pp. 707-715, 2014.

[53] C.-L. Wu, S.-D. Chen, J.-H. Yin, C.-S. Hwang, and D.-I. Yang, "Nuclear factor- $\kappa \mathrm{B}$-dependent sestrin2 induction mediates the antioxidant effects of BDNF against mitochondrial inhibition in rat cortical neurons," Molecular Neurobiology, vol. 53, no. 6, pp. 4126-4142, 2016.

[54] C. T. Shearn, D. S. Backos, D. J. Orlicky, R. L. SmathersMcCullough, and D. R. Petersen, "Identification of 5/ AMPactivated kinase as a target of reactive aldehydes during chronic ingestion of high concentrations of ethanol," Journal of Biological Chemistry, vol. 289, no. 22, pp. 15449-15462, 2014.

[55] S. J. Siegel, J. Bieschke, E. T. Powers, and J. W. Kelly, "The oxidative stress metabolite 4-hydroxynonenal promotes Alzheimer 
protofibril formation," Biochemistry, vol. 46, no. 6, pp. 15031510, 2007.

[56] Y. Kimura, Y. Mikami, K. Osumi, M. Tsugane, J.-I. Oka, and H. Kimura, "Polysulfides are possible $\mathrm{H}_{2} \mathrm{~S}$-derived signaling molecules in rat brain," The FASEB Journal, vol. 27, no. 6, pp. 2451-2457, 2013.

[57] Y. Kimura, Y. Toyofuku, S. Koike et al., "Identification of $\mathrm{H}_{2} \mathrm{~S}_{3}$ and $\mathrm{H}_{2} \mathrm{~S}$ produced by 3-mercaptopyruvate sulfurtransferase in the brain," Scientific Reports, vol. 5, article 14774, 2015. 


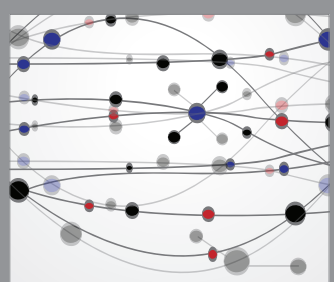

The Scientific World Journal
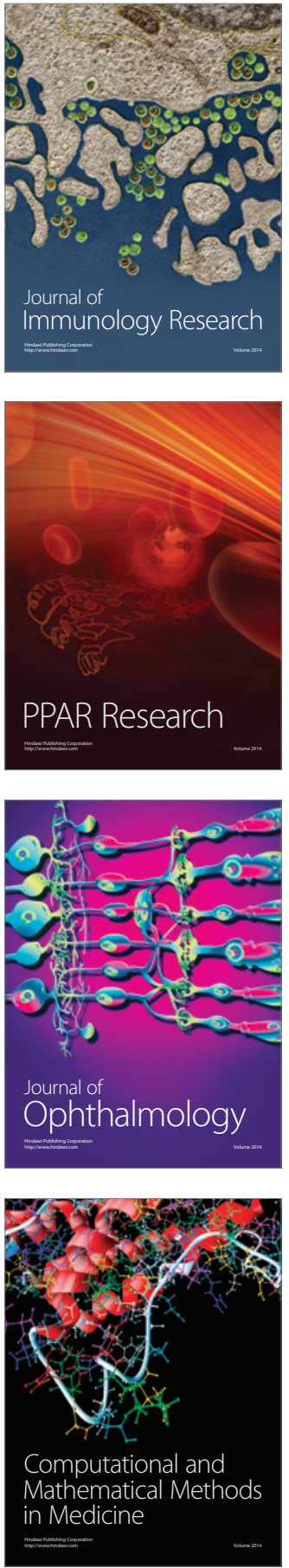

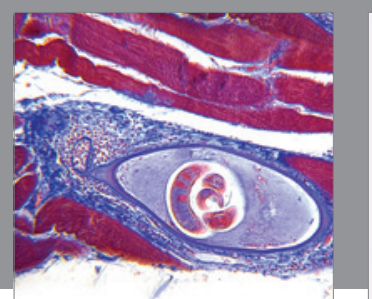

Gastroenterology Research and Practice

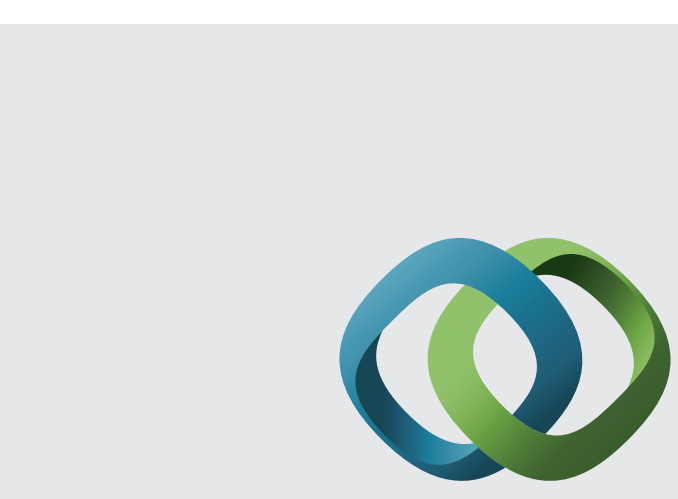

\section{Hindawi}

Submit your manuscripts at

http://www.hindawi.com
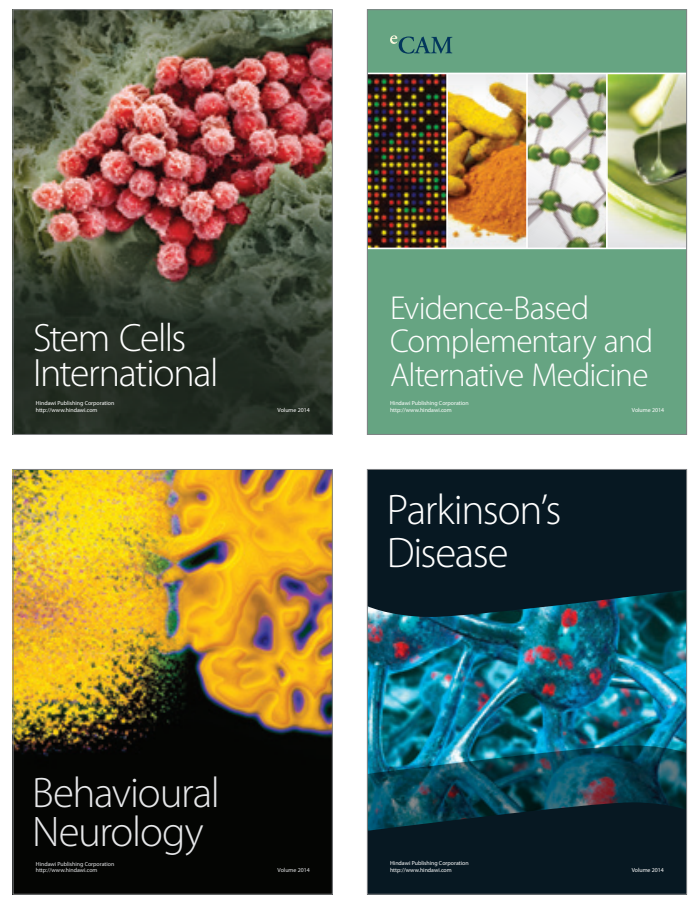
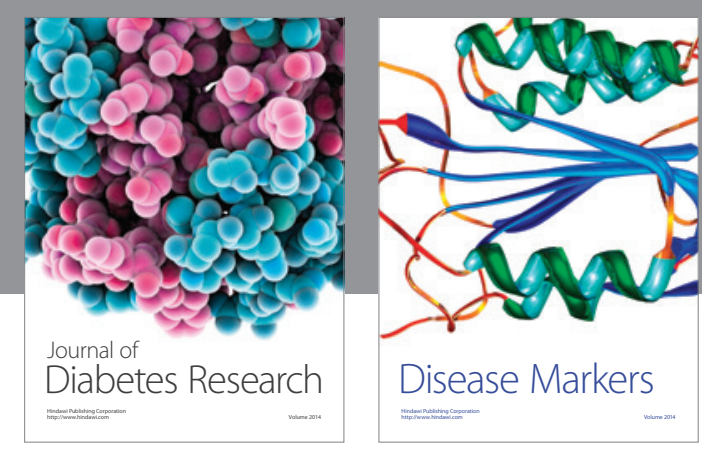

Disease Markers
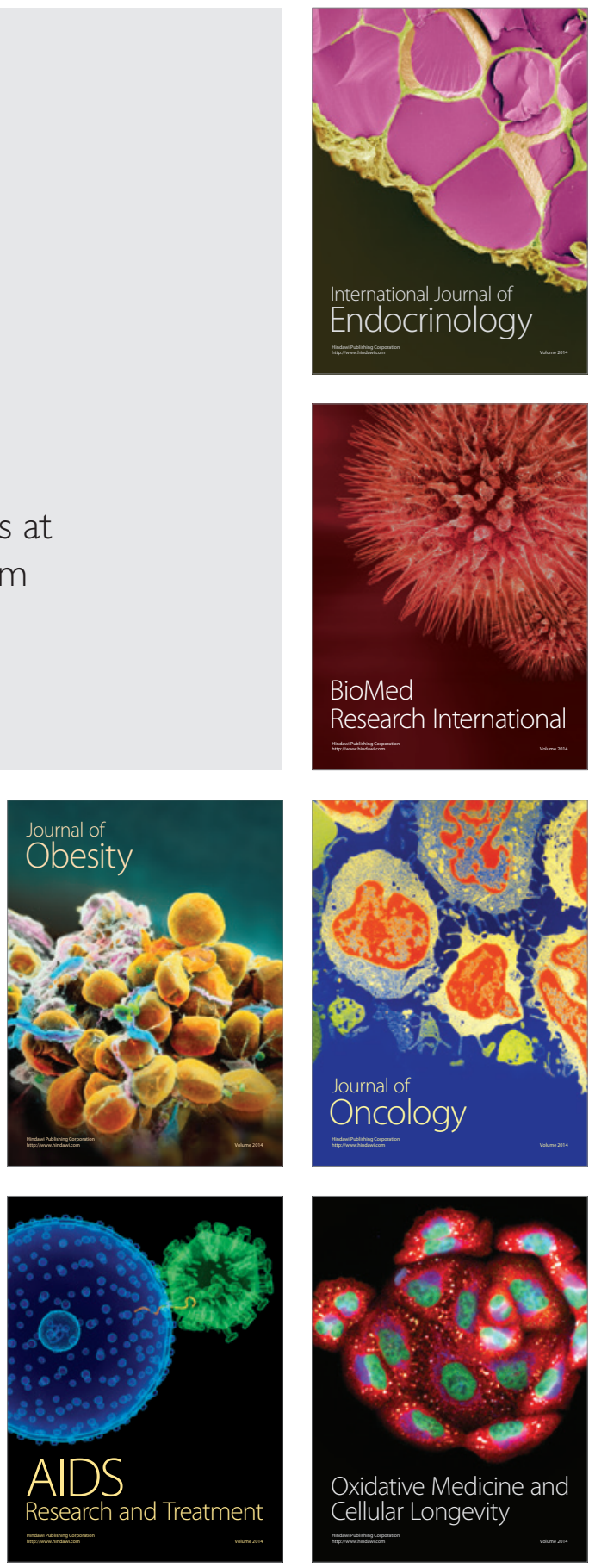